\title{
Electron tomography simulator with realistic 3D phantom for evaluation of acquisition, alignment and reconstruction methods
}

\author{
Xiaohua Wan ${ }^{\mathrm{a}, \mathrm{b}}$, Tsvi Katchalski ${ }^{\mathrm{b}}$, Christopher Churas ${ }^{\mathrm{b}}$, Sreya Ghosh ${ }^{\mathrm{b}}$, Sebastien \\ Phan $^{\mathrm{b}}$,AlbertLawrence ${ }^{\mathrm{b}, 1}$, Yu Hao ${ }^{\mathrm{a}}$, Ziying Zhou ${ }^{\mathrm{a}, \mathrm{d}}$, Ruijuan Chen ${ }^{\mathrm{e}, \mathrm{b}}$, Yu Chen ${ }^{\mathrm{a}}$, Fa \\ Zhang $^{\mathrm{a}}$, Mark H. Ellisman ${ }^{\mathrm{b}, \mathrm{c}}$ \\ ${ }^{a}$ Institute of Computing Technology, Chinese Academy of Sciences, Beijing, China. \\ ${ }^{b}$ National Center for Microscopy and Imaging Research, University of California, San Diego, USA; \\ ${ }^{\mathrm{c}}$ Departments of Neurosciences and Bioengineering, University of California, San Diego, USA. \\ ${ }^{\mathrm{d} B e i j i n g}$ Institute of Technology, Beijing, China. \\ ${ }^{\mathrm{e} S c h o o l ~ o f ~ E l e c t r o n i c ~ a n d ~ I n f o r m a t i o n ~ E n g i n e e r i n g, ~ T i a n j i n ~ P o l y t e c h n i c ~ U n i v e r s i t y, ~ T i a n j i n, ~ C h i n a . ~}$
}

\section{Abstract}

Because of the significance of electron microscope tomography in the investigation of biological structure at nanometer scales, ongoing improvement efforts have been continuous over recent years. This is particularly true in the case of software developments. Nevertheless, verification of improvements delivered by new algorithms and software remains difficult. Current analysis toolsdo not provide adaptable and consistent methods for quality assessment. This is particularly true with images of biological samples, due to image complexity, variability, low contrast and noise.

We report an Electron Tomography (ET) simulator with accurate ray optics modeling of image formation that includes curvilinear trajectories through the sample, warping of the sample and noise. As a demonstration of the utility of our approach, we have concentrated on providing verification of the class of reconstruction methods applicable to wide field images of stained plastic-embedded samples. Accordingly, we have also constructed digital phantoms derived from serial block face scanning electron microscope images. These phantoms are also easily modified to include alignment features to test alignment algorithms. The combination of more realistic phantoms with more faithful simulations facilitates objective comparison of acquisition parameters, alignment and reconstruction algorithms and their range of applicability. With proper phantoms, this approach can also be modified to include more complex optical models, including distance-dependent blurring and phase contrast functions, such as may occur in cryotomography.

\footnotetext{
keywords: electron; microscope; tomography; simulator; tilt; series; reconstruction; tracking; alignment; block face; inversion; curvilinear; warping;

${ }^{1}$ Corresponding author; email addresses: albert.rick.lawrence@gmail.com (Albert Lawrence)
} 


\section{Introduction}

\subsection{Electron Tomography}

Electron tomography (ET) enables detailed imaging of macromolecules, macromolecular complexes and subcellular assemblies within a whole cell (Frank, 2008). Three-dimensional (3D) reconstructions of those structures are typically generated from a set of projection images acquired at different sample orientations by tilting the specimen around one (Marabini, et al., 1997) or more axes (Mastronarde, 1997; Phan et al., 2016). In spite of advances in microscopy technology and software analysis high-resolution 3D localization and visualization in situ of individual proteins and assemblies of macromolecular complexes within cells and tissues remain a difficult goal to achieve. At present molecular dynamics (MD) simulations can yield short time period and atomic scale resolution of macromolecules, but in-situ and biologically relevant information is lacking. A considerable volume of research into ET is largely directed toward improving spatial resolution and closing the resolution gap with single particle cryoEM. Alternatively, simulations of dynamics by MD and other methods may be used to provide verification. For example, cryotomography at the shorter spatial scales relates biological processes at longer spatial scales to changes at the level of large molecules and micromolecular assemblies. Furthermore, in order to improve our knowledge of the structure and function of macromolecules in biological systems, ET is used today to provide 3D information over spatial scales ranging from macromolecules (nanometers) to cells and tissues (micrometers to millimeters).

Research into wide field ET at longer spatial scales is coupled to advances in fluorescence light microscopy. With the advent of many new genetic probe-based or click chemistry-based correlated light and electron microscopy (CLEM) labeling strategies(Martell et al., 2012; Ngo et al., 2016; Shu et al., 2011), it is desirable to facilitate production of higher qualityET data sets from plastic-embedded specimens(Frank, 2008; McEwen et al., 2008). In contrast to fluorescent label-based light microscopy, which provides a more limited view of the distribution of only the fluorescently labeled constituents, ET allows the simultaneous visualization of all the intricate complexity of biological structures around the cellular or subcellular domain under investigation.

\subsection{Problems with ET}

However, compared to procedures for high-resolution light microscopy, ET remains a relatively complicated method in both the cases of plastic embedded and frozen sections. For example, to image tissues or thicker regions of epoxy-embedded cells, semi-thin sections must generally be produced by ultramicrotomy and then imaged from many different sample orientations, yielding a set of digital EM images. These images must be processed through several steps to derive the 3D volume for use in further analyses. High-quality reconstruction results from ET are hampered by several major obstacles: First, tilt angles in ET owing to technical and material characteristics are generally limited to a range within $\pm 70^{\circ}$ due to scatteringof the primary electron beam. The resulting limitation in tilt and inability to collect high angle projections is often referred to as themissing wedgeproblem(Frank, 2008); Second, reconstructions are affected by the distortions that result from the imperfect optics of the electron microscope and the sample warping due to radiation exposure damage (Luther, 2006). 
Additionally, electron micrographs are quite noisy and low contrast, especially under low dose collection conditions or when employing higher electron beam voltage.

The assumption of travel along straight-line paths as in X-ray tomography is not true in an electron microscope, as focusing is performed by means of magnetic fields. Figures illustrating this effect appear in various publications(Phan et al., 2012; Reimer and Kohl, 2008). Because electrons travel in curvilinear trajectories under the influence of magnetic fields it is generally not possible to align properly all regions in large images using 2D deformation. Observationally, deviations on the order of 50 pixels and more at the periphery of $8 \mathrm{k}$ x 8k pixel images taken at high sample tilt angles may occur. This deviation comes from objects at different distances from the focal plane moving in ways that are not predictable from models based on straight ray transforms. This problem must be solved by considering the geometric implications of curvilinear electron trajectories in the formation of an image. In addition, lens aberrations, sample mass loss and consequent warping confounds the problem of curvilinear trajectories. These problems may be addressed together by calculating effective trajectories through the sample.

Until recently ET data sets were typically recorded along one or two curves in the sphere of available directions. Examples of this are the so-called single tilt (rotation of the sample around an axis perpendicular to the optical axis) and conical tilt series (rotation of the sample around the optical axis, (Lanzavecchia et al., 2005)).Observationally, reconstruction from single tilt data can give very different appearances in the $x y$ plane perpendicular to the optical axis and the other planes that contain the optical axis. The detection of gradients that are perpendicular or nearly perpendicular to several beam directions is hampered if there are large gaps between projection directions.This is improved with double tilt acquisitions, and the recent adaptation of multiple rotations in the plane perpendicular to the optical axis using four or more tilt series. Although the problem of homogeneous gradient detection may be addressed by data acquisition along well-distributed sets of projection, the issue of determining the nature of specific improvements still remains.

A recent publication reports that the reduction of beam damage and accurate alignment makes possible the acquisition and processing of large numbers of projections at various orientations(Phan et al., 2016). Specifically,artifacts in planes perpendicular to the tilt axis,which are associated with single tilt series acquisitions,can be greatly reduced. However, the magnitude of theimprovements in detection of density gradients provided by additional projection data may be diminished by smoothing effects due to slight misalignments of these merged tilt series.

\subsection{Purpose of this work}

Numerous phenomena impact image data acquisition and the quality of 3D reconstructions from EM images. Digital reconstruction methods must address the quality and resolution issues posed by the particular nature of EM imaging. Accordingly, we have constructed a classical model of EM imaging and assembled a library of instrument model parameters that correspond to image acquisition. In order to complete the correspondence, we also require a realistic phantom object. We reasoned that arealistic digital phantom along with a faithful ray optics model of EM imaging would provide a basis for objective comparisonsbetween various 
tomographic reconstruction strategies. For example, such a tool would be expected to provide a platform for tests ofnoise and artifact suppression methods as well as different alignment and reconstruction processes currently in use. Furthermore, we can address the problem of testing reconstruction algorithms across spatial scales ranging from microns to nanometers.

Toprovide usefulrealism, we constructed a digital representation based on volume imaging data obtained by serial block face scanning electron microscopy (SBEM). Block face imaging method generates relatively high resolution representations of biological structures, and provides an independent means of constructing phantoms.

Because the data is derived from a series images of the specimens' block face as its' surface layer is repetitively removed, reconstructions based on this techniqueare free of many inherent deficiencies of phantoms built fromvolumes acquired by ET owing to TEM optics. Specifically, there are no artifacts associated to a missing wedge, beam helicity or discretization associated with data from a limited number of observational directions. Furthermore, reconstructions correspond well to the electron densities within the original object. A reconstruction from a SBEM data could therefore be employed as a realistic source of data. In this work, we report on methods employing a SBEM volume, and the production of synthetic projection images that capture many of the relevant elements of TEM.

Simulation of transmission electron microscope images of biological specimens is implemented in a number of software packages for electron microscopy such as IMAGIC(van Heel et al., 1996), Xmipp(Bilbao-Castro et al., 2004), SPIDER (Shaikh et al., 2009), EMAN2 (Tang et al., 2007), Bsoft (Heymann and Belnap, 2007) and TOMToolbox(Nickell et al., 2005). Usually a virtual model of a biological specimen is created using highly regular 3D figures (phantoms) such as spheres, ellipsoids, cubes, and cylinders(Bilbao-Castro et al., 2004) or tubular domains within a matrix having slab-geometry(Chen et al., 2014). The phantoms are generally tailored to test specific features of reconstruction schemes. Such piecemeal schemes result in these objects being over-simplifications of experimental data in electron tomography. Simulated TEM images are then computed by projecting the 3D specimen. The projection model adopted is most commonly a simple straight-line projection model, which does not account for the distortions introduced by helical electron trajectories in the objective lens and beam-induced sample warping. Aside from effects due to sampling limitations, aberration and defocus, these are the most important sources of degradation of resolution in ET reconstructions.We also note that phantom investigations that are restricted to 2D neglect some of the most evident resolution problems in EM tomography, such as the differing appearances of reconstruction along sections other than the one perpendicular to the optical axis, as detailed above.

Because the purpose of this work is to develop simulations of heavy metal stained plastic embedded samples, we have chosen to model beam interactions in the simplest possible manner consistent with previous publications in this arena (Mastronarde, 1997).

Depending on sample characteristics the contrast mechanism may be associated with either of two mechanisms, phase contrast or amplitude contrast. Phase contrast is typical of thin unstained samples, where the coherence of the electron beam is maintained, a region typically coined as the weak phase-object approximation (WPOA). The contrast transfer function (CTF) is dominated by spherical aberration 
and defocusing distance a combination of effects causing the CTF to typically oscillate till other envelope effects take dominance. Amplitude contrast on the other hand, is characteristic of stained or thick samples where coherence is lost. While amplitudes are combined in phase contrast, intensities are combined in amplitude contrast. We have concentrated on intensity contrast in our initial investigations.

First principle accounts starting with the Schrödingerequation may be found in the materials sciences literature relating to image formation from thick samples of ordered materials (Kirkland, 2010). So-called multislice methods for computer based models of image formation also afford a potential bridge between the theoretical settings of cryotomography and thick disordered samples (Elbaum et al., 2014; Scott et al., 2012). In principle, the mathematical assumptions and physical approximations that reduce the more complicated multiple scattering-based model to a single scattering model are available in several publications. The main issue is the proper form of the point spread function, which models blurring effects (Kazantsev et al., 2010; Kirkland, 2010; Klukowska, 2008; Marabini et al., 2016; Oton et al., 2012).Given that these models describe the elastic scattering portion of sample-beam interactions, there is still the problem of inelastic scattering as described by(Reimer and Kohl, 2008). In effect, some of the electrons interact with the electrons in the sample, yielding some energy in the process. These shifts to lower energy give rise to chromatic aberrations, which, in turn blur the image. A further complication is due to defocus. Because we are dealing with samples which have a considerable spatial extent in the plane perpendicular to the optical axis, we must also deal with defocus (Reimer and Kohl, 2008)(Lawrence et al., 2012). Blurring due to defocus can accentuate the chromatic aberration effects and lead to the problem of separation. Finally, a first principles study of beam effects, must also deal with local magnetic fields. This would necessitate inclusion of a magnetic field term in addition to the potential term(Kasper and Hawkes, 1996). We include this effect as a rotation of the beam(Lawrence et al., 2006).

For thick and wide field samples, it has been shown(Lawrence et al., 2006), that neglect of errors in backprojection, due to helicity can be large compared to the extent of point spread functions due to defocus and chromatic aberration. Errors in the periphery and at large tilts can be many tens of pixels, exceeding the distance at which point spread functions drop off into background noise levels and so helicity has been included in the model.

Accordingly, this work describes an ET simulator together with a realistic 3D phantom with digital objects, called ET-SPEC, i.e. Electron Tomography- $\underline{\text { Simulator }}$ and Phantom for Evaluation and Comparison initially designed for wide field investigations of thick plastic imbedded, heavy metal stained samples.

\section{Mathematical Model}

The methods employed in this study include both a realistic digital phantom and a detailed microscope model. Both are necessary to provide detailed comparisons of reconstruction software systems on a common basis, as well as ground truth information for evaluation of specific reconstruction schemes. We describe the acquisition and preprocessing of the digital phantom, our microscope model, simulated data acquisition, sample effects, and marker placement in subsequent sections. Phantom and simulator image formation are schematically presented in figure 1 below. 


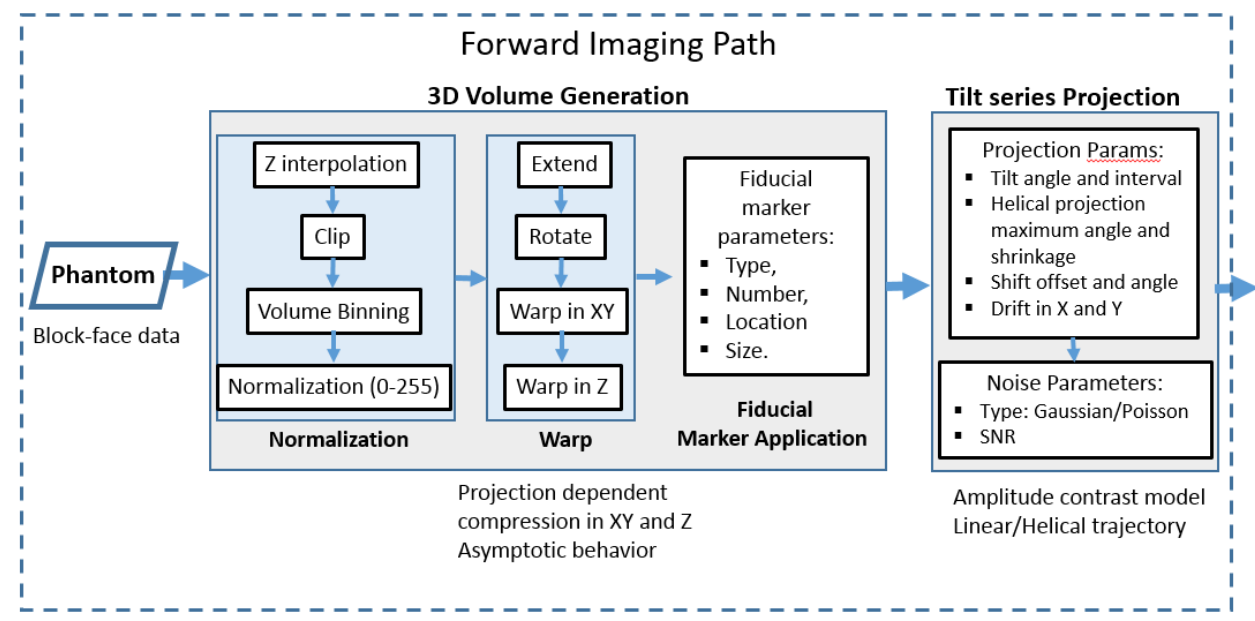

Figure 1: Phantom and simulator image formation schematic. After phantom generation from SBEM additional processing is done to the phantom, including adding noise and fiducial markers. Finally, projections are generated based on desired illumination scheme.

\subsection{Digital Phantom}

A SBEM reconstruction of brain tissue extracted from a mouse cerebellum was used as the starting point. The data was generated using a Zeiss Merlin scanning electron microscope equipped with an ultramicrotome (Gatan 3View system). Thickness of serial cuts taken to remove material between sequential image acquisition was $30 \mathrm{~nm}$. Data was collected with the Gatan Digital Micrograph software as 16 bit images in a proprietary dm3 format, then converted to Tiff images. The data set consisted of total of 100 micrographs, each containing $12 \mathrm{k}$ x $8 \mathrm{k}$ pixels. Each image was collected by detecting the backscattered electrons, the microscope operating at an acceleration voltage of $1.9 \mathrm{kV}$. During the acquisition process, Digital Micrograph automatically inverted the contrast tomake images resemble TEM micrographs. In a first computational treatment, each slice was scaled independently to bring to common mean and standard deviation. The data was then pre-aligned using cross-correlation procedures to ensure a smooth optical flow in the $\mathrm{Z}$ direction, at which point each voxel would represent a cuboid portion of the specimen of dimension $1 \mathrm{~nm} \times 1 \mathrm{~nm}$ x $30 \mathrm{~nm}$. Due to limitations imposed by the microtome, the Zresolution of the data does not correspond to the sample. In effect, each voxel represents an integration over 30 pixels in the $\mathrm{Z}$ direction due to the volume sampled by the backscattering. One effect is to smooth the gradients in this direction, as compared to downsampling, so despite the loss of resolution, the statistics of gradient magnitudes along all three axes should be mutually consistent. Because our investigation is directed toward verification of algorithm performance rather than validation of our reconstruction with respect to ground truth on the physical level, this should afford a fair test of algorithm performance.

Following the adjustments performed at the instrument we performed two additional treatments with the object to make the density and spatial scales homogeneous. First, we assigned pixel values in the electron micrographs to the specimen metal density by matching the maxima and variances of the density histograms. The unknown constants are chosen to extend the histograms over the numerical range of the sensors, and outliers in the tails are assigned new values representing the average of surrounding 
pixels.

We used 3D volume data of brain tissue showing neuron bundles at $1 \mathrm{~nm} \mathrm{x}, \mathrm{y}$ sampling (pixel size). Figure 2, shows internal volume $X Y, X Z$ and $Y Z$ cross sections. The many circular structures are axonal processes of neurons (Cerebellar parallel fibers) while the $\mathrm{Y}$ shaped middle structure is a large branching dendritic process of a Purkinje neuron. Markers were placed on the top and bottom surface of the phantom with marker size of 9 and $7 \mathrm{~nm}$ respectively and numbering to a total of 220. For this investigation, warping and padding were not applied. The marker positions were noted and used to assist in the alignment step of the reconstruction. The projection data is MRC image format compatible and can be displayed using iMOD as shown in in figure 4 in the next section.

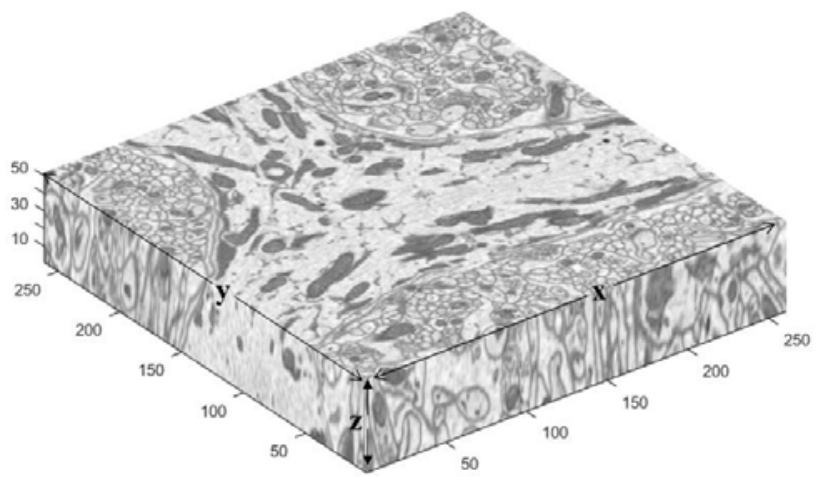

Figure 2: Internal volume cross sections of 3D data obtained by clipping a larger volume. The top $X Y$ and side $Z X$ and $Z Y$ facets are shown. The preservation of fine structure within this $3 D$ phantom will be investigated in detail.

For quick analysis, we worked with a sample volume of 360x360x75 pixels. Compared to a real ET situation, the present phantom data has different edge conditions than an actual sample in an electron microscope. We may make various choices to make edge conditions correspond, for example, we could augment the data by padding to make sure all trajectories intersecting the sample are utilized. This produces a density discontinuity on its borders, but has the advantage of precise constraints, which can be applied outside the boundaries, particularly in iterative reconstructions. For cases where we want to simulate the usual conditions, particularly with filtered backprojection the object can be extended by mirroring,i.e., reflecting across the boundaries.

The procedure above produced a realistic digital phantom, to which we then apply transforms representing sample rotation and warping, a beam trajectory model and various noise models. This allows the testing of alignment procedures using features representing gold beads and other markers that may be inserted into the digital object. We discuss this process below and provide descriptions for the electron beam model, sample warping, and fiducial markers steps. 


\subsection{Beam Model}

The underlying physical model for ET is a classical single scattering process from a beam with initial intensity $I_{i}$ in an object with density $u(X, Y, Z)$. Note: We generally use uppercase for object coordinates and lower case for image (projection) coordinates. We assume a family of non-intersecting trajectories, $\Gamma$, in three dimensions

$\left(\gamma_{1}(s), \gamma_{2}(s), \gamma_{3}(s)\right)$, parameterized by path length parameter $s$,

$$
\Gamma(s)=\left(\gamma_{1}(s), \gamma_{2}(s), \gamma_{3}(s)\right)
$$

with beam intensity $I_{f}$ at exit point $\left(X_{f}, Y_{f}, Z_{f}\right)$ given by the exponential of integral along the trajectory $\Gamma_{\left(X_{f}, Y_{f}\right)}$ through the exit point:

$$
I_{f}(x, y)=I_{i} \exp \left(-\int u\left(\Gamma_{(x, y)}(s) d s\right)\right)
$$

We assume that the scattering probability is a constant multiple of the density along the trajectories, up to a constantK,

$$
\log \left(I_{f}\right)=\log \left(I_{i}\right)-\int K u\left(\Gamma_{(x, y)}(s)\right) d s
$$

Precise determination of the initial intensity and the scattering intensity generally require calibrations that are not commonly performed. We therefore must choose constants in such a way to minimize the effects of outliers, and make the object more or less homogeneous statistically. Generally, the log transform is applied to laboratory data and this tends to diminish the effect of different choice of constants.

The electron trajectories are assumed to be helical with a twist angle rate $\mu$ and convergence rate $\kappa$ along the $Z$-axis.

$$
\left[\begin{array}{l}
X(t) \\
Y(t)
\end{array}\right]=(1-\kappa t)\left[\begin{array}{cc}
\cos (\psi) & \sin (\psi) \\
-\sin (\psi) & \cos (\psi)
\end{array}\right]\left[\begin{array}{l}
X_{i} \\
Y_{i}
\end{array}\right](4)
$$

Each point in the electron beam trajectory may be parameterized by $t$ and its position in the plane of origin $\left(X_{i}\right.$ and $\left.Y_{i}\right)$. The first two coordinates of exit point $\left(X_{f}, Y_{f}, Z_{f}\right)$ correspond to the beam projection onto the camera, where $t=Z_{i}-Z$ and the twist angle given by $\psi=\mu t$. We set the initial value of $Z$ to be the sample thickness and the final value of $\mathrm{Z}$ to be zero. In practical situation, the condition $\kappa \Delta Z \ll 1$ must be satisfied. Typical parameters for the twist and shrinkage rates are, $\mu=0.00005, \kappa=0.0004$. The arc length parameter is obtained by integrating the arc length element along this curve, and the exit point may be calculated by straightforward application of the trajectory formula.Because the degree of twist is small, using Z-coordinate instead of arc length introduces a negligible error.

Noise can also be added to the trajectories. Either Poisson noise or Gaussian noise can be added where the SNR level can be selected.In these investigations simulated noise was not added and the exiting noise is that which was already present in the phantom, so as to keep the focus on the parameters under investigation. Similarly, for the same reason, warping was not included as well. 


\subsection{Specimen warping}

We use a simple model to describe the specimen warping - the bending and twisting of sample from its initial shape, which results from the electron beam radiation.Sample warping, also termed sample distortion, is attributed to strains created by inhomogeneous mass loss (de Jonge et al., 2010; Duan et al., 2014; Suzuki, 2002). The fraction of distortion may be modeled by a convergence to a fixed constant $\sigma_{\infty}$ as the number of exposures increases.

$$
\sigma_{\tau}=\left(1-e^{-n_{\theta} \tau}\right) \sigma_{\infty}
$$

The parameter $\tau$ is a scaling factor controlling the rate of convergence and $n_{\theta}$ is a positive parameter which can represent time or exposure number. In the remainder, we will differentiate the warping when it occurs in the $X Y$ plane (noted with an index $\perp$ ) from warping in $Z$ direction (noted with an index $\|$ ).

In the following, we use the center $\mathrm{O}$ of the volume as the coordinates origin to define the warping transformations. Typical parameters for warping distortion are $\tau=0.1$ and $\sigma_{\infty}=0.1$.

\subsubsection{Compression in $X Y$}

To describe the compression of the specimen within the $X Y$ plane, we use the following expression:

$$
(\widetilde{X}, \tilde{Y})=\left(1-\sigma_{\tau \perp}(X, Y)\right)
$$

\subsubsection{Compression/Warping along $Z$}

.. To describe the warping along $Z$, we use the following expression:

$$
\tilde{Z}=\left(1-\sigma_{\tau \|}\right) Z+\sigma_{\tau \|} P(X, Y)
$$

Where $P$ is a polynomial function. In our investigation, we use a second-degree polynomial.

Sample warping that occurs in ET is not as simple as our model implies; local distortion may depend on the staining itself. According to our observations, areas in the sample with less stain tend to be more prone to deformations. This may be compensated by using higher degree polynomials in all three coordinates; the problem here is that the most common alignment practice employs gold markers on the surfaces of the object, and as a consequence the markers may not be well placed to detect complex warping.

To summarize the last two sections, the impact of linear vs. helical trajectory on the projections and the impact of sample warping are demonstrated in Figure 3.1 and Figure 3.2 respectively. As shown in Figure 3.1 by the difference image, the impact of helicity is small in the center of the image and increases at the periphery. In addition, as the projection is generated the helical beam samples locations rotated in $X Y$ causing the final projection to seem blurred compared to the linear projection. The impact of helicity is expected to be stronger for tilted samples as the $Z$ span is larger in this case.As shown in Figure 3.2, warping causes geometrical distortion-shrinkage of the sample which is accumulated with dose (axis number) it can involve simple 
shrinkage in $x y$ as shown in (e) for $0^{\circ}$ tilt and more complex geometrical distortion as shown in (g) at $60^{\circ}$ tilt, where non-symmetric Z-dependent warping takes effect.
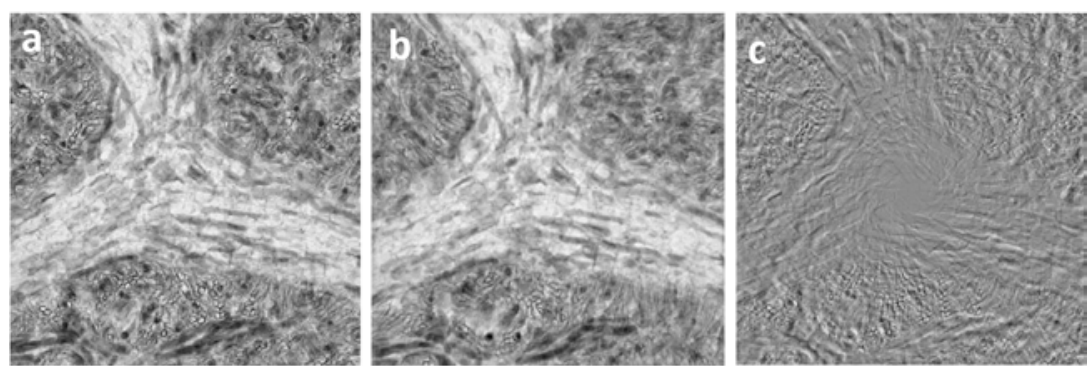

Figure 3.1:Helical vs. Linear projection image.Projections obtained at $0^{\circ}$ tilt angle.(a) linear vs. (b) helical trajectory. (c) is a difference image. From difference image, the magnitude of helicity increases radially, maximal at the image periphery. The helical beam projection as shown in (b) samples in way which causes an appearance of blurring relative to the linear projection (a). Helical trajectory parameters: Twist angle rate $\mu=0.008$ and convergence rate $\kappa=0.001$
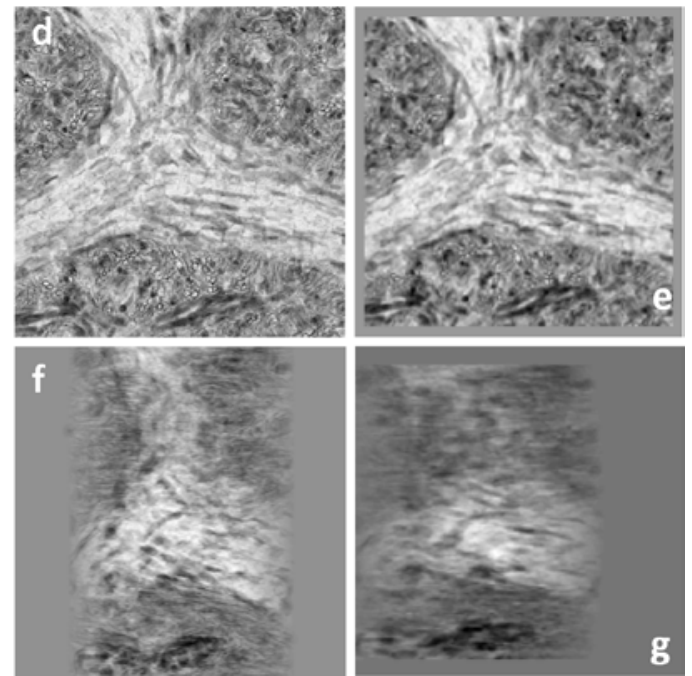

Figure 3.2: Warped vs. un-warped projection image. The result of warping for $0^{\circ}$ and $60^{\circ}$ are shown in the top and bottom row respectively. While warping at $0^{\circ}$ is exemplified as shrinkage of dimension along the periphery of the object, a more complex behavior is observed at $60^{\circ}$ tilt where non-symmetric Zdependent warping takes effect. Warping distortion parameters: $\tau=0.5$ and $\sigma_{\infty}=0.5$.

\subsection{Fiducial markers}

We may also add electron dense particles on each side of the sample to mimic the gold markers commonly used for alignment purpose.

\subsubsection{Density profile of the markers.}

The simplest choice is to define a characteristic function of a ball of radius $a$ :

$$
\chi_{\alpha}(X)=\operatorname{sign}((1,1,1)-\alpha X)
$$

and 


$$
u=u_{\max } \chi_{\alpha}\left(X-X_{i}\right)
$$

Alternative choices are possible to describe the 3D density variations associated with fiducial markers. For instance, a Gaussian profile can be used:

$$
u=u_{\max }\left(1-\rho \exp \left(-\alpha\left\|X-X_{i}\right\|^{2}\right)\right)
$$

This model could be extended to simulate diffraction, as markers used (such as gold beads) often exhibit semi-crystalline structure. In this case the appropriate function would be a sum of derivatives of the Gaussian profile function. Another option would be:

$$
u=u_{\max }\left(1-\frac{\rho}{1+\alpha\left\|\boldsymbol{X}-\boldsymbol{X}_{\boldsymbol{i}}\right\|^{2}}\right)
$$

Here $\boldsymbol{X}_{\boldsymbol{i}}$ represents the center of marker $i, u_{m a x}$ is the density corresponding to the electron opaque material, $a$ and $\rho$ are parameter characteristic of the marker size $(\sim 1 / \sqrt{a}$ ) and electron density respectively. These parameters can be modulated in particular to test detection algorithms on situations when the markers are barely noticeable.

\subsubsection{Marker location}

The markers may be randomly placed on the external surfaces of the specimen or in a regular arrangement that makes systematic reprojection errors more visible. One issue is whether to embed the markers in the layer closest to the exterior, and apply the warping transform to the center of the marker or to tether the marker to a point on one of the $X Y$-surfaces, and calculate the motion of the marker as the sample is warped. The more realistic method is the latter, as there may be significant motion of the marker centers for more extreme warping. In this work, we apply the simpler method, because we assume small markers, and minor sample warping.

When generating any synthetic projection, we may apply the warping transformations on the naked specimen first, and then add the untouched markers at the right locations. These locations are calculated so a marker bead is always tangent to the same specimen point. We note here $M_{k}$ and $T_{k}$ the locations of the center of marker kand its contact point on the specimen; $\widetilde{\mathrm{M}}_{k}$ and $\widetilde{\mathrm{T}}_{k}$ are the equivalent points after the warping occurred.

The two conditions:

$$
\left\{\begin{array}{l}
\boldsymbol{M}_{k}-T_{k}=r n_{k} \\
\widetilde{\boldsymbol{M}}_{k}-\widetilde{\boldsymbol{T}}_{k}=r \tilde{n}_{k}
\end{array}\right.
$$

are satisfied, where $=1 / \sqrt{a}$ corresponds roughly to the marker radius, and $n_{k}$ and $\tilde{\mathrm{n}}_{\mathrm{k}}$ are the exiting normal to the specimen evaluated at the contact points before and after the warping.

The two surfaces $S_{i}(X, Y)$ and $S_{f}(X, Y)$ limiting the specimen volume in the $Z$ direction are defined by $Z=Z_{i}$ and $Z=Z_{f}$ before the warping, and $\widetilde{Z}_{i}=$ $\mathcal{M}\left(X, Y, Z_{i}\right)$ and $\tilde{Z}_{f}=\mathcal{M}\left(X, Y, Z_{f}\right)$ after the warping $\mathcal{M}$. Given this the direction of the exiting normal to the initial surface is given by the cross product of the two tangent directions to the surface of the sample, evaluated at the contact positions, e.g., for markers on the top and bottom respectively: 


$$
\begin{gathered}
n_{\text {top }}=\left(1,0, \frac{\partial \widetilde{Z_{i}}}{\partial X}\right) \times\left(0,1, \frac{\partial \widetilde{Z_{i}}}{\partial Y}\right) \\
n_{\text {bottom }}=-\left(1,0, \frac{\partial \widetilde{Z}_{f}}{\partial X}\right) \times\left(0,1, \frac{\partial \widetilde{Z}_{f}}{\partial Y}\right)
\end{gathered}
$$

\section{Methods}

To mimic a series of EM images, the first step is to include the markers as small spheres by substituting the digital representation of each marker for the original data. Similarly, data for other included structures, such as mitochondria can be substituted. In the next steps of producing each simulated EM image, the modified phantom volume is first warped incrementallybefore each projection. Subsequent to the warping, the sample is rotated into position. Finally,the generalized radon transform as described above, yields a curvilinear projection on a 2D plane. In the current investigation,we use a multiple axis series protocol corresponding to usual experimental procedure for the rotations. We may first specify tilt axis tethered to the sample, which may be parallel to the $Y$-axis. We then apply a rotation in the $X Y$ plane followed by a rotation around the new axis. In order to achieve a symmetrical and uniform distribution of viewing angles we rotate and tilt by rational fractions of $2 \pi$.

\subsection{Specimen stage orientation}

Corresponding to the discussion above, there are two preferred rotations in the reference coordinates of the microscope around which the sample can be oriented. The sample can first be rotated around the microscope optics axis $\left(\mathbf{u}_{\mathbf{z}}\right)$, which is usually done manually. The sample can then be tilted in a direction perpendicular to the tilt axis (for instance $\mathbf{u}_{\mathbf{Y}}$ ); this is usually done automatically with the goniometer.

\subsubsection{Rotation around the optical axis}

A double tilt series corresponds to two tilt series with the sample pre-rotatedat $0^{\circ}$ and $+90^{\circ}$.

$$
X \rightarrow \mathcal{R}_{\phi, u_{z}}(X)
$$

\subsubsection{Sample tilt with the goniometer}

A tilt series is usually a sequence of goniometer rotation from $-60^{\circ}$ to $+60^{\circ}$ every $2^{\circ}$.

$$
X \rightarrow \mathcal{R}_{\theta, u_{Y}}(X)
$$

\subsubsection{Slash/Backslash effects of the sample stage}

Depending on the quality of the hardware, positioning of the sample can be quite imprecise. We can model this by introducing additional rotational $\left(\delta_{\mathrm{R}, 1}, \delta_{\mathrm{R}, 2}\right)$ and translational $\left(\delta_{\mathrm{T}}\right)$ noisein the transformation product describing the stage motion.

According to the stages discussed above, the combined stage effect is expressed as follows: 


$$
\left[\begin{array}{l}
X_{S} \\
Y_{S} \\
Z_{S}
\end{array}\right]=\left[\begin{array}{ccc}
\cos \left(\theta+\delta_{R, 1}\right) & 0 & \sin \left(\theta+\delta_{R, 1}\right) \\
0 & 1 & 0 \\
-\sin \left(\theta+\delta_{R, 1}\right) & 0 & \cos \left(\theta+\delta_{R, 1}\right)
\end{array}\right] \cdot\left[\begin{array}{ccc}
\cos \phi\left(\phi+\delta_{R, 2}\right) & -\sin \left(\phi+\delta_{R, 2}\right) & 0 \\
\sin \left(\phi+\delta_{R, 2}\right) & \cos \left(\phi+\delta_{R, 2}\right) & 0 \\
0 & 0 & 1
\end{array}\right]\left[\begin{array}{c}
\tilde{X} \\
\tilde{Y} \\
\tilde{Z}
\end{array}\right]+\delta_{T}
$$

\subsubsection{Projection of the markers}

In an alternative use of our simulation and modeling software, markers need not be inserted into the digital representation of the phantom. Rather, auxiliary files containing original and projected marker positions can be carried forward into the reconstruction. The use of the projected marker positions may be used to eliminate tracking steps in 3D reconstruction, while the use of both would eliminate the necessity for alignment steps, with approximations to the projection maps being calculated directly from 3D and 2D position files. This would give additional flexibility in evaluating various reconstruction algorithms.

\subsection{Code development}

All the above models have been implemented in an interactive graphical tool named ET-SPEC by Xiaohua Wan. ET-SPEC has been developed following a modular approach, using $\mathrm{C}$ as the programming language, QT as the graphic user interface (GUI) design library and OpenGL as the 3D graphics library. The choice of language and libraries makes ET-SPEC highly portable to most operating systems where those libraries are available.

ET-SPEC includes four parts: 3D volume constructed, tilt-series projection, alignment and reconstruction, allowing interactive creation of $3 \mathrm{D}$ model and tiltseries. The users can construct a 3D volume based on original SEM data and add gold markers on both sides of 3D volume by choosing optional parameters according to their needs. ET-SPEC is available free for non-commercial use, to obtain a copy please send an email to: etspec@ncmir.ucsd.edu. In the future, we plan to make all code available as a repository within the CRBS organization on Github (http://github.com).

\subsection{Reconstruction}

In order to demonstrate the use of our simulation software and digital phantom in an evaluation study we employed the reconstruction package TxBR(Lawrence et al., 2006). To provide high-quality reconstructions, the projection images must be aligned accurately. In TxBR, this is done with a non-linear bundle adjustment scheme that jointly optimizes the micrograph registration to the final volume and nonlinear projection maps corresponding to electron trajectories. The computed trajectories are implicitly parametrized by means of polynomial maps approximating the projection maps. Approximation is by means of 2nd degree polynomials in the voxel positions. In the laboratory setting TxBR can account for a sizable amount of sample warping, optical distortion, and non-linear trajectories, which makes it a good candidate to align and reconstruct multiple large-field tilt series(Phan et al., 2012).

TxBR employs a filtered backprojection (FBP) routine. Once the alignment step is completed, filtered densities are backprojected in the final 3D reconstruction via the computed projection. We employed a Shepp-Logan filter applied to the simulated 
data in to correct the point-spread function generated by the backprojection(Natterer and Wubbeling, 2001).

\section{Results}

As a demonstration of the potential of both phantom and simulation tool we explored the impact of several multi-tilt acquisition configuration and reconstructions on general image quality and resolution of the final reconstructed volume. It is well known that single tilt acquisitions suffer from degraded resolution and visibility of the reconstructed volume, especially along the $z$ (optical axis) direction, due to partial angular sampling, also known as the missing wedge. Double tilt acquisition is frequently suggested as common practice to improve resolution and visibility of features. Using a realistic phantom with fine detail in all $X, Y$ and $Z$ directions and the simulation tool described above we evaluated quantitatively the improvements obtained with the following strategies:

(a) Single-axis vs. dual-axis, 4-axis, 8-axis and 16-axis series acquisitions.

(b) Impact of angular increment on visibility of features.

The results of the above simulations and more of this kind, will assist in investigating the tradeoffs typically encountered in experimental tomography settings.

\subsection{Multiple axis series projection}

Multiple axis series were generated using the microscope simulator. Each axis series spanned tilt angles from $-60^{\circ}$ to $+60^{\circ}$ with increment of $\pm 2^{\circ}$ typically but also of $\pm 1^{\circ}$ and $\pm 10^{\circ}$. Various axis series were generated from single-axis up to 16-axis series, including dual-axis series with rotation angles $\alpha=0^{\circ}, 90^{\circ}$, 4-axis series $\alpha=$ $0^{\circ}, 45^{\circ}, 90^{\circ}, 135^{\circ}, \quad 8$-axis $\quad$ series $\quad \alpha=$ $0^{\circ}, 22.5^{\circ}, 45^{\circ}, 67.5^{\circ}, 90^{\circ}, 112.5^{\circ}, 135^{\circ}, 157.5^{\circ}$ etc.Projections were generated with helical electron beam trajectories. The helicity was defined using two parameters, rotation angle $\mu$ and shrinkage $\kappa$ as detailed in section 3.2.1. Values $\mu=0.00005, \kappa=0.0004$ used are typical for experimental EM scanners corresponding to a few degrees of beam rotation as it passes the sample. The generated 8-axis series are shown in Figure 4. The 8-axis angles $\alpha=0^{\circ}, 22.5^{\circ}, 45^{\circ}, 67.5^{\circ}, 90^{\circ}, 112.5^{\circ}, 135^{\circ}, 157.5^{\circ}$ are shown in sub figures a-h respectively, all taken at the same tilt angle $15^{\circ}$. A similar 16-axis series was also generated. In these investigations simulated noise was not added and the exiting noise it that which originated with the phantom, so as to keep the focus on the parameters under investigation. Similarly, for the same reason, warping was not included as well. We have also neglected various types of blurring which could be described by point spread functions in our simulation. This is because the software under test is not designed to deal with blurring beyond the effects of miscalculation of electron trajectories and sample warping effects. Furthermore, our observations on wide field samples generally show that the spatial extent of blurring is less than the displacement of features due to helicity (Lawrence et al., 2006).

\subsection{Final reconstruction evaluation}

Because we have the original 3D volume and can calculate the exact position of each marker per the helical electron trajectory, alignment and reconstruction methods in ET can be evaluated based on ET-SPEC. In previous methods, alignment performance could be indirectly evaluated by the quality of reconstructions. The 
problem with this approach is that there are also effects of reconstruction methods on the reconstructed results that make it difficult or impossible to disentangle the various effects of alignment and reconstruction. In ET-SPEC, we may calculate the exact position of each marker directly and compare with the position of each marker obtained using different alignment methods developed both in our lab and in other labs. For the reconstruction of the various tilt series we used Transform Based Bundle Adjustment and Reconstruction algorithm - TxBR(Lawrence et al., 2006).

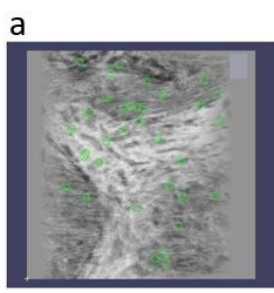

e

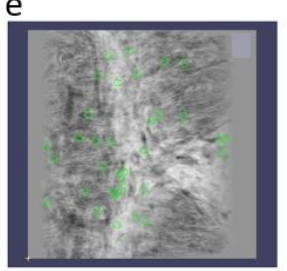

b

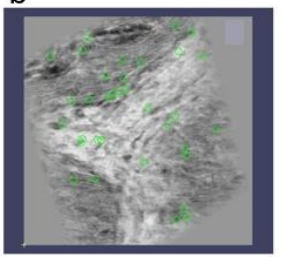

f

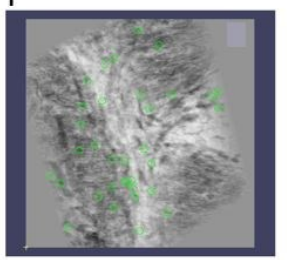

C

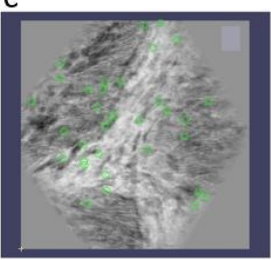

g

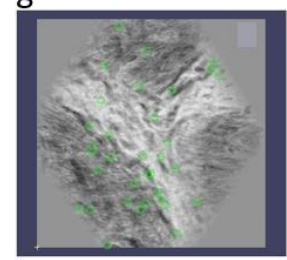

d

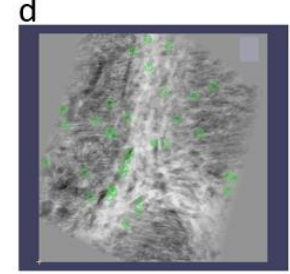

h

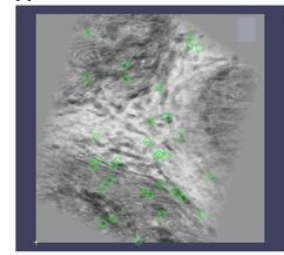

Figure 4:Generated 8-axis seriesprojectiondata. The 8-axis angles $\alpha$

$=0^{\circ}, 22.5^{\circ}, 45^{\circ}, 67.5^{\circ}, 90^{\circ}, 112.5^{\circ}, 135^{\circ}, 157.5^{\circ}$ are shown in sub figures a-h, all taken at the same tilt angle $15^{\circ}$. The green circles are the location of the marker beads used for alignment. The projections were displayed using iMOD.

4.2.1. Evaluation of reconstructions from single-axis vs. dual-axis, 4-axis, 8axis and 16-axis series acquisitions

First, we compared the impact of multiple axis acquisition on artifact suppression. The simulated gold beads are a good starting point as they are highly attenuating objects in a uniform background and come in various spatial locations. Figure 5 compares the original sample-phantom to results of single-axis, dual-axis, 4-axis, 8axis and up to 16-axis series reconstruction. As a general note, to facilitate comparison between the images, all were normalized in a way as to have zero mean and unit standard deviation:

$$
I_{n}(X, Y, Z)=\frac{I_{o}(X, Y, Z)-M}{S}
$$

where $I_{n}$ and $I_{0}$ are the normalized and original intensity and $M$ and $S$ are the mean and standard deviation of the volume.

As can be seen in figure 5(a-f) of $X Y$ cross section, the directional bright streaks projecting from the beads in single-axis and double-axis are very dominant, especially across lines containing several adjacent beads. Four-axis series alleviates the artifact, and adding more axes adds incremental improvement both in reducing streak artifacts near the beads and improving the uniformity of the background area as well. Artifacts in $X Z$ and $Y Z$ are reduced as well. The observed results are consistent with improved sampling isotropy with increasing axis number(Natterer and Wubbeling, 2001). In figure 5(h-i) we see in addition, $X Z$ and $Y Z$ plots of a single- 
axis and 8-axis reconstruction. The known hourglass artifact in the $Y Z$ plane is visible for the single axis while suppressed for the eight-axis. In both cases elongation of the cylindrical beads is also observed and can be attributed to sampling anisotropy.

To facilitate feature preservation comparison and to quantify the trends from one tilt-axis reconstruction to another, we generated 3D gradient volumes from the reconstructed volumes. Figure 6(a) and (b) show $X Y$ cross sections of the original volume and sixteen-axis reconstructed volume. The red vertical profile corresponds to the profile at the location of the vertical (red) line. The horizontal (blue) profile corresponds to the profile along the horizontal (blue) line. It is evident from the profiles that features are lost, so there is a tradeoff, between resolution fidelity and artifact suppression. We discuss this further below.
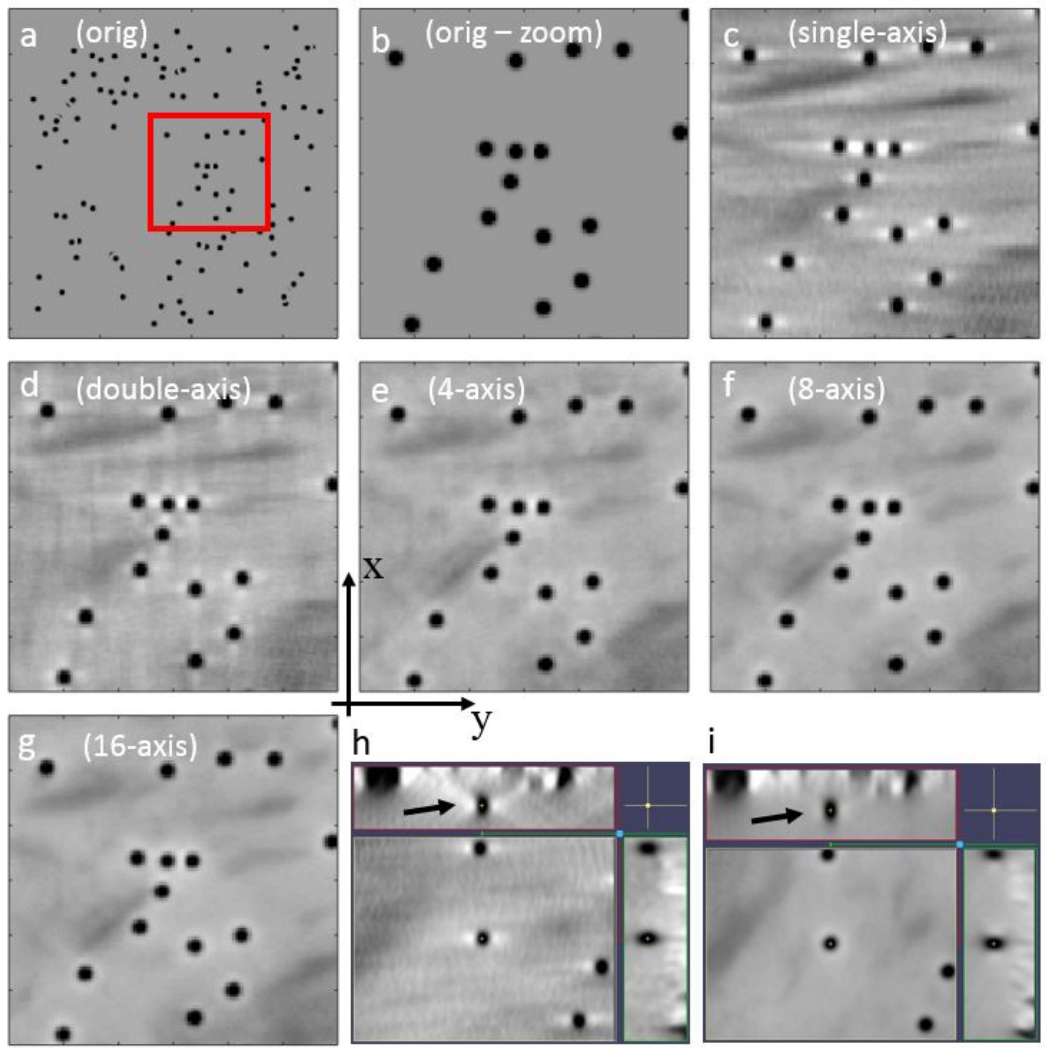

i

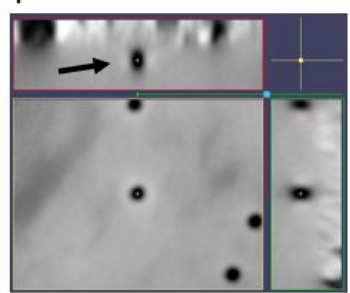

Figure 5: Bead containing $X Y$ section of the phantom. Top row from left (a) original phantom (b) zoomed area of original phantom within the red rectangle. Followed images are reconstructions of the zoomed area. The tilt axis is along the yaxis. Top right (c) single-axis reconstruction. Middle row from left (d) dual-axis (e) 4-axis and (f) 8-axis reconstruction. Bottom row from left (g) 16-axis reconstruction. (h) iMOD XYZ plot centered on a bead, of a single-axis reconstruction (i) iMOD $X Y Z$ plot centered on a bead, of an 8-axis reconstruction. In (h) and (i) Center panel is $X Y$, top is $Y Z$ and right is $X Z$ cross section. The hourglass artifact is marked with the black arrow in (h) and it's absent, noted in (i). All images displayed using the same window-center 

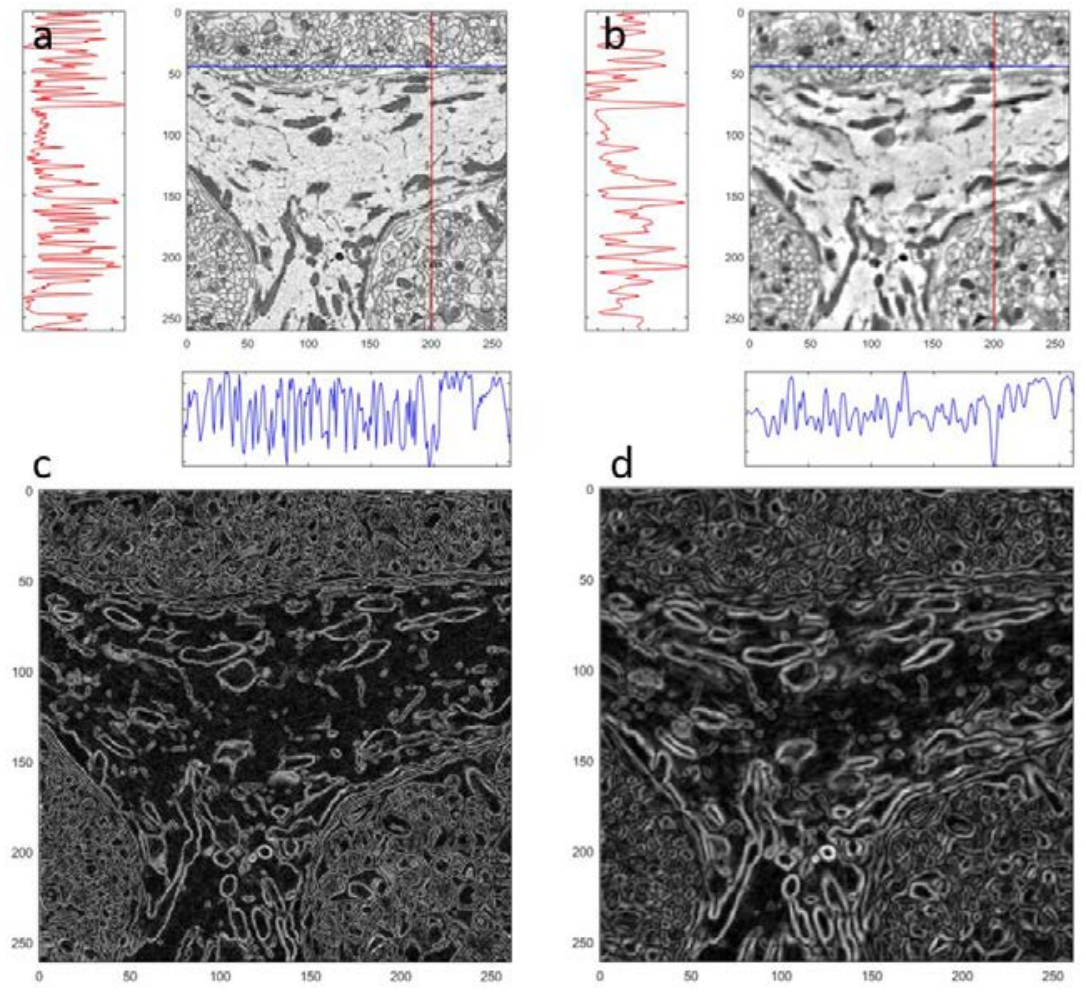

Figure 6: (a) Original volume $X Y$ cross section and (b) reconstructed 16-axis series volume $X Y$ cross section. The vertical (red) and horizontal (blue) profiles are also presented. These were taken at the positions marked by the vertical and horizontal lines. Bottom row plots are gradient-magnitude, $\|G\|, X Y$ cross section plots for the (c) original and (d) reconstructed 16-axis series volume. Gradient plots facilitate comparison of feature preservation between the various axis-series reconstructions.

The gradient along $X, G_{x}$ is defined to be the average of the difference of neighboring pixels of the volume image $I\left(X_{i}, Y_{j}, Z_{k}\right)$ along $X$, i.e.

$$
G_{x}\left(X_{i}, Y_{j}, Z_{k}\right)=\frac{I\left(X_{i+1}, Y_{j}, Z_{k}\right)-I\left(X_{i}-1, Y_{j}, Z_{k}\right)}{2},
$$

and similarly, for the other directions. The gradient magnitude is defined as the gradient vector norm,

$$
\|G\|=\left\|G_{x}\left(X_{i}, Y_{j}, Z_{k}\right), G_{y}\left(X_{i}, Y_{j}, Z_{k}\right), G_{z}\left(X_{i}, Y_{j}, Z_{k}\right)\right\|
$$

Figure 6(c) and (d) plot the $X Y$ cross section of gradient magnitude for the original volume and the 16-axis volume. The loss of structural detail is easily seen in these images as thickening or loss of fine features.

The deviation of the final reconstructed volume relative to the original is estimated in a total-variation like measure where we define the total variation

$$
\begin{aligned}
\Delta G=\sum_{i, j, k}\left\|\left(G_{X, O}\left(X_{i}, Y_{j}, Z_{k}\right), G_{Y, O}\left(X_{i}, Y_{j}, Z_{k}\right), G_{Z, O}\left(X_{i}, Y_{j}, Z_{k}\right)\right)\right\| \\
-\left\|\left(G_{X, R}\left(X_{i}, Y_{j}, Z_{k}\right), G_{Y, R}\left(X_{i}, Y_{j}, Z_{k}\right), G_{Z, R}\left(X_{i}, Y_{j}, Z_{k}\right)\right)\right\|
\end{aligned}
$$


where $O$ and $R$ symbolize the Original measure and n-axisReconstruction respectively and will be used as a measure of resolution preservation relative to the original ground truth volume. The feature restoration trend as you increase the number of tilts can be seen in figure 7 .

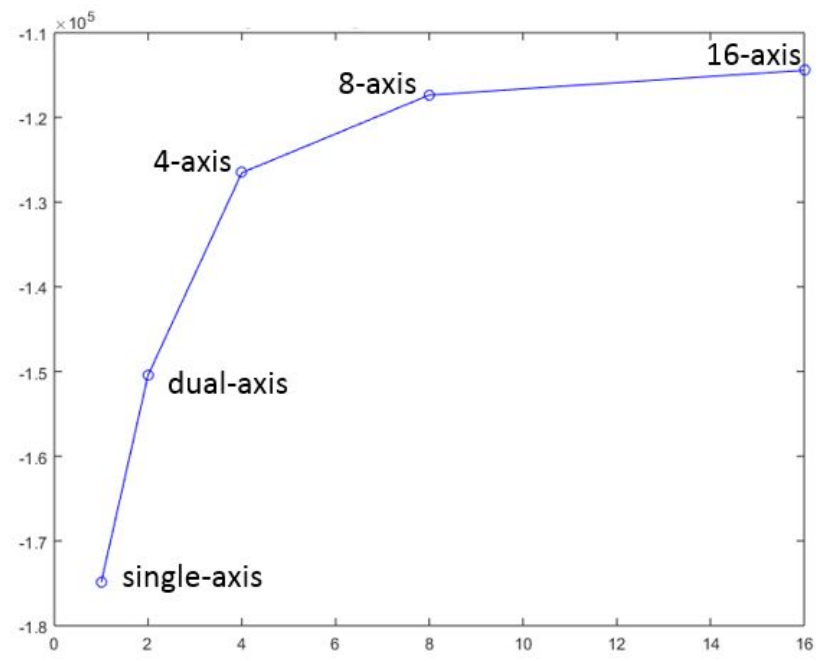

Figure 7: Gradient total variation as a function of the number of axis series taken, comparing the original volume and the reconstructed volumes as a function of the number of axis series taken.

As we increase the number of axes we see that the agreement between phantom and reconstruction improves but gradually with increasing axis number reaching maximum improvement at the largest number of axes. In summary, it can be seen that the addition of axis series supports artifact suppression. For resolution improvement, there is a slowly continuing improvement in resolution but the largest improvement occurs over the first few axis series.

\subsubsection{Impact of angular increment on visibility of features}

We investigated the impact of angular increment on feature visibility. For this purpose, we looked into the four-axis reconstruction series once with angular increment of $\pm 1^{\circ}$ and once with $\pm 10^{\circ}$, and we investigated the impact on various reconstructed cross sections of the volume.

Figures8 and 9 show $X Z$ and $Y Z$ cross sections respectively of the original volume vs. the other two reconstructed volumes with the middle slice for the dense sampling and the right most slice for the low sampling increment. The top part of the figure schematically illustrates the angular sampling density in the cross section below it. As can be seen there is considerable degradation in the $X Z$ and $Y Z$ cross section detail at $\pm 10^{\circ}$ increment. The arrow marked features are ones captured in the high angular 
increment sampling acquisition but are lost in the low angular increment sampling. Such an analysis will be useful in setting the expectation of feature visibility given a set acquisition method.

A natural extension of the demonstrated phantom and simulation would be to test various alignment and reconstruction methods using simulated projection data. Those tests can include various publiclyavailable reconstruction packages such as iMOD and perhaps for comparing FBP vs. iterative reconstruction methods such as SIRT.

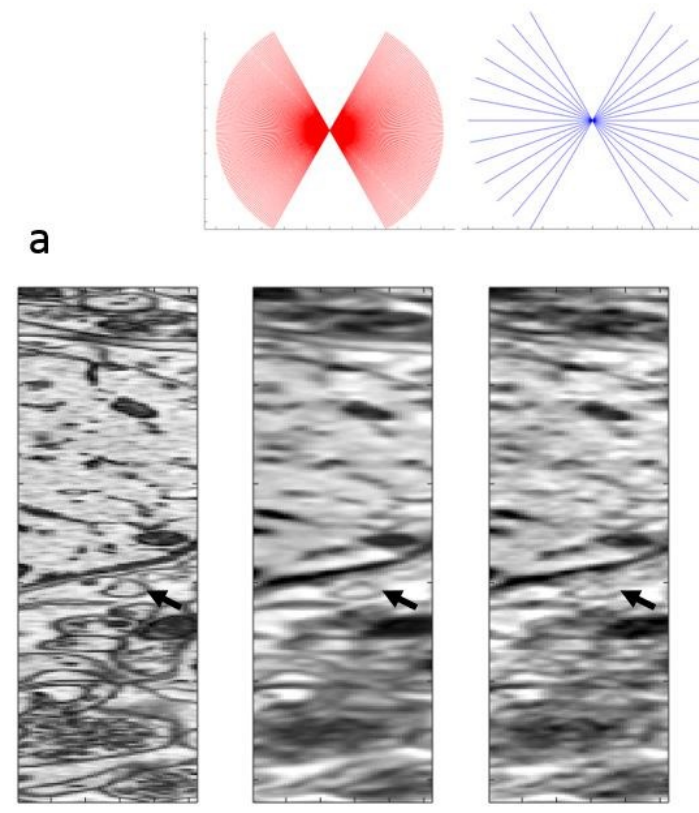

Figure 8: $X Z$ cross section of the (left) original volume (middle) $\pm 1^{\circ}$ increment 4 -axisacquisition reconstructed volume and (right) $\pm 10^{\circ}$ increment 4-tilt acquisition reconstructed volume. The black arrow mark clearly visible difference but additional differences are also noted throughout the image. The top row schematically illustrates the angular sampling density in the cross sections directly below it. 


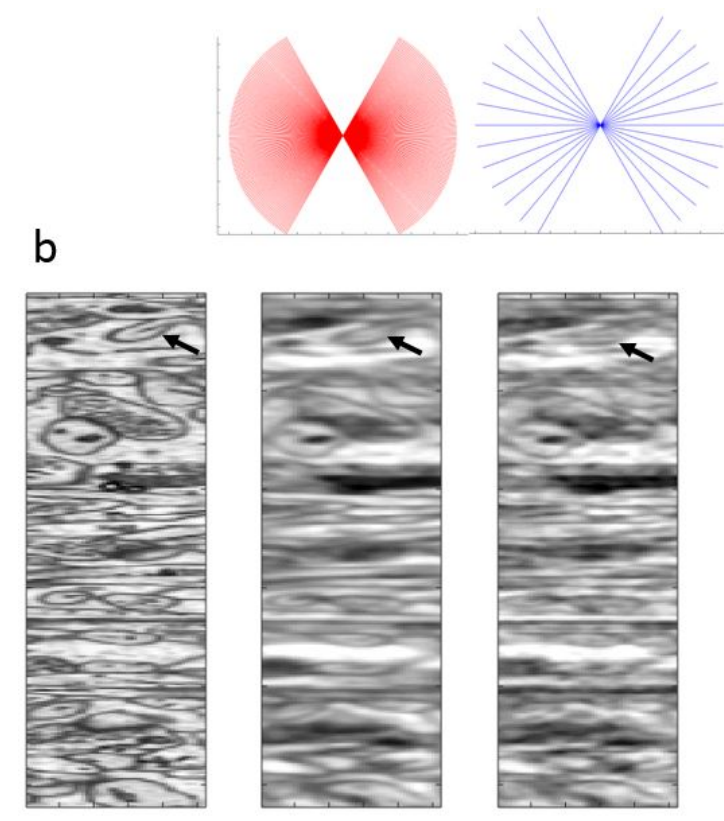

Figure 9: $Y Z$ cross-section of the (left) original volume (middle) $\pm 1^{\circ}$ increment 4 -axis acquisition reconstructed volume and (right) $\pm 10^{\circ}$ increment 4-axis acquisition reconstructed volume.

\section{Discussion and Conclusion}

We have demonstrated the application of a realistic phantom and electron microscope model to the evaluation of acquisition parameters for electron microscope tomography and application of the TxBR reconstruction code. In particular, we have demonstrated the effects of increasing the number of projections on resolution and reconstruction artifact in a multiple axis series data acquisition scheme. Increasing the number of projections with a more uniform coverage of the available $X Y$ rotations is shown to reduce artifact, but also tends to smooth out the peaks in graphs of line profiles in the $X Y$ plane. This shows up in comparisons between the original data and the final reconstruction. On the other hand, increasing the number of tilt angles in rotations around an axis in an $X Y$ plane appears to yield improvements in resolution when the gradient in the $Z$ direction is brought into consideration. It also appears that improvements in resolution in the $Z$ direction counterbalance smoothing in the $X Y$ planes when multiple tilt series are employed in the reconstruction.

The finding regarding $X Y$ rotations is somewhat unexpected, but indicates that the alignment and reconstruction algorithms may not be performing according to a simple model of resolution. At least two factors may be significant in the $X Y$ plane smoothing. First, the high frequency cutoff in the Shepp-Logan filter(Natterer and Wubbeling, 2001)could induce undue smoothing in the $X Y$ plane. Previous work tuned this cutoff for single tilt axis data sets, but further work is necessary for multiple tilt axes. In particular, the cutoff in the filter should be moved toward higher 
spatial frequencies. Second, because the alignment step is set to approximate electron trajectories by order two polynomials, and the beam model assumes helical trajectories, it is possible that errors in the backprojection may be responsible for the unexpected smoothing in the $X Y$ plane.Helical trajectories are much better approximated by order three polynomials.In summary, it appears that we have identified two trends which work against each other, that is the expected improvement in the $Z$ direction and the unexpected smoothing in the $X Y$ planes. Further possible factors are loss of resolution in the $Z$ direction due to missing tilts at high angles, and blurring due to defocus at high tilts, but more work is necessary to untangle the consequent effects.

In any experimental endeavor, both verification and validation serve essential roles. Verification is the proof that our methods work as intended, in this case, the computations are done correctly. Validation, on the other hand is the proof that our models correspond in a measurable sense to physical reality. In prior work(Phan et al., 2016), based on actual laboratory data, we observed both the smoothing and reduction of artifacts associated with additional $X Y$ rotations. Unfortunately, with the prior work, ground truth was not available. In this work, we have performed a necessary verification test from ground truth that gives a strong indication that the problems observed are not due to physically invalid algorithms. This is further indicated by the reconstruction of gold markers, which are well known to be spherical high-density objects. Iterative method can preserve the form of the markers without distortion or artifacts(Phan et al., 2016). Whether iterative methods are relatively insensitive to $3 \mathrm{D}$ alignment methods remains to be established. Although iterative methods may give better resolution than filtered backprojection, each step in the iteration requires a full backprojection, and many tens of iterations are necessary for satisfactory convergence.

\section{Future Work}

The present study has indicated the need for follow-up work directed toward the improvement of the alignment step in the ET workflow. Beyond this, validation (as opposed to verification) depends on independent information concerning the structure of a sample at spatial scales not commonly accessed by ET. At present our phantom data originates in the observation of a sample preparation through destructive removal of very thin serial sections from stained samples fixed in plastic. Other techniques may be used for validation. For example, the spatial scales in X-ray microtomography nearly overlap the spatial scales in ET (Mizutani and Suzuki, 2012), and with modest improvements in resolution the information from X-ray microtomography can be collected non-destructively before sectioning the sample for ET. Some degree of validation is also available at the small spatial scales from molecular modeling. Structural determination of macromolecular assemblies, up to the size of whole viruses can be achieved with current molecular modeling techniques. This data can also be used in verification studies

In the visible light domain, we have fluorescence microscopy. Although the spatial scales probed by fluorescence techniques are longer than the spatial scales in ET, fluorescence microscopy gives location of specific molecular structures down to the nanometer range, and with special modification of the basic technique, small 
markers may be deposited around the fluorescence site (Schellenberger et al., 2014). With the usual procedure of sample preparation, this gives further information that can be fed into the ET workflow.

Besides the distortions associated with sample warping and helical electron trajectories considered in ET-SPEC, an accurate model of image formation in ET should include the effects of aberrations such as defocusing, astigmatism and spherical aberration as well as beam-sample interactions. Multislice methods afford more advanced treatments of beam-sample interaction. A multislice model may be included in iterative methods for 3D reconstruction, but such computational methods require major computational resources.

Application of Fourier transform methods may reduce the order of computation of the forward model to the theoretical lower limit (Kirkland, 2010). Following Kirkland's example in the case of forward models, we should also consider the magnitude of the computational problemfor 3D inversion.Compressive sensing has received considerable attention in the X-ray and ET communities as a means for reducing the computational magnitude of the inversion problem. This, however, pushes the problem back to the calculation of representative frames in that one major obstruction to applying the results of studies of compressive sensing is the sheer magnitude of the data generated in many biological studies (Leary et al., 2013; Thomas et al., 2013). The massive sample size and high dimensionality of Big Data introduce computational and statistical challenges, including scalability, noise accumulation, spurious correlation, incidental endogeneity and measurement errors. These challenges require new computational and statistical paradigm (Fan et al., 2014). Because of these problems, current approaches to the problems associated with compressive sensing may not be computationally feasible in our present case. Nevertheless, theoretical studies of the linear problem associated with the classical Radon transform are available in the X-ray case.

Singular value and Toeplitz matrix decompositions may reduce the magnitude of the computational problem. The issue here is that we mustinvert a generalized Radon transform as described in our model above (Cai et al., 2010; Chandrasekaran et al., 2008; Lawrence et al., 2006).The singular value transform for the standard ray transform is well-known (Maass, 1987), but one of the remaining problems is finding good approximations for the generalized (curvilinear) ray transform. One of the more promising approaches would be based on Toeplitz matrices. Toeplitz matrices have all values along each diagonal constant, and most matrix computations involving $N \times N$ Toeplitz matrices are of order $N^{2} \log (N)$. Note the number of operations in standard matrix multiplication is of order $N^{3}$. This fact has spurred the application of Toeplitz methods in a wide range of scientific applications (Ye and Lim, 2016).

The FFT is used to reduce many computations involving structured matrices such as Toeplitz to the order of $N\left(\log ^{k}(N)\right)$ operations. Toeplitz systems may in fact be solved in $O\left(N^{2} \log ^{2} N\right)$ via the use of displacement rank(Bitmead and Anderson, 1980). It has also been shown that every $N \times N$ matrix can be written as the product of $2 N+5$ Toeplitz matrices(Ye and Lim, 2016). This has implications for reconstruction methods, also. The magnitude of 3D reconstruction for large samples requires large computational resources. Furthermore, integral formula methods for reconstruction via multislice methodsare not well developed(Klukowska, 2008). Accordingly,high quality reconstruction would require the application of iterative 
techniques, compounding the computational problem. Thus, the application of Toeplitz matrix methods deserves further study.

\section{Acknowledgements}

This work was funded by the International Community Foundation under the Sun Yat Sen Fund for international Cultural Exchange and Collaboration. The authors thank Dr. R. Ramachandra and Prof. J. M. Carazo for helpful discussions.

\section{References}

Bilbao-Castro, J.R., Sorzano, C.O.S., García, I., Fernández, J.J., 2004. Phan3D: Design of biological phantoms in 3D electron microscopy. Bioinformatics 20, 3286-3288.

Bitmead, R.R., Anderson, B.D., 1980. Asymptotically Fast Solution of Toeplltz and Related Systems of Llnear Equations *. Linear Algebra Appl. 34, 103-116.

Cai, J.-F., Candes, J.E., Shen, Z., 2010. A Singular Value Thresholding Algorithm for Matrix Completion 20, 1956-1982.

Chandrasekaran, S., Gu, M., Sun, X., Xia, J., Zhu, J., 2008. A Superfast Algorithm for Toeplitz Systems of Linear Equations. SIAM J. Matrix Anal. Appl. 29, 1247-1266.

Chen, D., Goris, B., Bleichrodt, F., Mezerji, H.H., Bals, S., Batenburg, K.J., de With, G., Friedrich, H., 2014. The properties of SIRT, TVM, and DART for 3D imaging of tubular domains in nanocomposite thin-films and sections. Ultramicroscopy 147, 137-148.

de Jonge, N., Sougrat, R., Northan, B.M., Pennycook, S.J., 2010. Three-Dimensional Scanning Transmission Electron Microscopy of Biological Specimens. Microsc. Microanal. 16, 54-63.

Duan, S., Chen, S., Yu, P., Li, M., Zhang, M., Chien, K., 2014. Optimization of TEM Sample Preparation to Reduce the Overlapping of TEM Images.

Elbaum, M., Wolf, S.G., Houben, L., 2014. Cryo-scanning transmission electron tomography of biological cells. Nat. Methods 11, 423-428.

Fan, J., Han, F., Liu, H., 2014. Challenges of Big Data Analysis. Natl Sci Rev 1, 293-314.

Frank, J., 2008. Electron Tomography: Methods for Three-Dimensional Visualization of Structures in the Cell. Springer Science \& Business Media.

Heymann, J.B., Belnap, D.M., 2007. Bsoft: Image processing and molecular modeling for electron microscopy. J. Struct. Biol. 157, 3-18.

Kasper, E., Hawkes, P.W., 1996. Principles of Electron Optics. Academic Press.

Kazantsev, I.G., Klukowska, J., Herman, G.T., Cernetic, L., 2010. Fully threedimensional defocus-gradient corrected backprojection in cryoelectron microscopy. Ultramicroscopy 110, 1128-1142.

Kirkland, E.J., 2010. Advanced computing in electron microscopy, Advanced Computing in Electron Microscopy: Second Edition. Springer Science \& Business Media, New York.

Klukowska, J., 2008. TR-2008011: Contrast Transfer Function Correction in Electron Microscopy.

Lanzavecchia, S., Cantele, F., Bellon, P.L., Zampighi, L., Kreman, M., Wright, E., Zampighi, G.A., 2005. Conical tomography of freeze-fracture replicas: A 
method for the study of integral membrane proteins inserted in phospholipid bilayers. J. Struct. Biol. 149, 87-98.

Lawrence, A., Bouwer, J.C., Perkins, G., Ellisman, M.H., 2006. Transform-based backprojection for volume reconstruction of large format electron microscope tilt series. J. Struct. Biol. 154, 144-167.

Lawrence, A.F., Phan, S., Ellisman, M., 2012. Electron tomography and multiscale biology. Lect. Notes Comput. Sci. (including Subser. Lect. Notes Artif. Intell. Lect. Notes Bioinformatics) 7287 LNCS, 109-130.

Leary, R., Saghi, Z., Midgley, P.A., Holland, D.J., 2013. Compressed sensing electron tomography. Ultramicroscopy 131, 70-91.

Luther, P.K., 2006. Sample shrinkage and radiation damage of plastic sections. Electron Tomogr. Methods Three-Dimensional Vis. Struct. Cell 17-48.

Maass, P., 1987. The x-ray transform: singular value decomposition and resolution. Inverse Probl. 3, 729-741.

Marabini, R., Oton, J., Pereiro, E., Conesa, J.J., Chichon, F.J., 2016. XTEND : Extending the depth of field in soft X-ray tomography. pp. 2-3.

Martell, J.D., Deerinck, T.J., Sancak, Y., Poulos, T.L., Mootha, V.K., Sosinsky, G.E., Ellisman, M.H., Ting, A.Y., 2012. Engineered ascorbate peroxidase as a genetically encoded reporter for electron microscopy. Nat. Biotechnol. 30, 1143-8.

Mastronarde, D.N., 1997. Dual-axis tomography: an approach with alignment methods that preserve resolution. J. Struct. Biol. 120, 343-352.

McEwen, B.F., Renken, C., Marko, M., Mannella, C., 2008. Chapter 6 Principles and Practice in Electron Tomography, 1st ed, Methods in Cell Biology. Elsevier Inc.

Mizutani, R., Suzuki, Y., 2012. X-ray microtomography in biology. Micron 43, 104115.

Natterer, F., Wubbeling, F., 2001. Mathematical Methods in Image Reconstruction, Book. Society for Industrial and Applied Mathematics.

Ngo, J.T., Adams, S.R., Deerinck, T.J., Boassa, D., Rodriguez-Rivera, F., Palida, S.F., Bertozzi, C.R., Ellisman, M.H., Tsien, R.Y., 2016. Click-EM for imaging metabolically tagged nonprotein biomolecules. Nat. Chem. Biol. 12, 1-10.

Nickell, S., Förster, F., Linaroudis, A., Del Net, W., Beck, F., Hegerl, R., Baumeister, W., Plitzko, J.M., 2005. TOM software toolbox: Acquisition and analysis for electron tomography. J. Struct. Biol. 149, 227-234.

Oton, J., Sorzano, C.O.S., Pereiro, E., Cuenca-Alba, J., Navarro, R., Carazo, J.M., Marabini, R., 2012. Image formation in cellular X-ray microscopy. J. Struct. Biol. 178, 29-37.

Phan, S., Boassa, D., Nguyen, P., Wan, X., Lanman, J., Lawrence, A., Ellisman, M.H., 2016. 3D reconstruction of biological structures: automated procedures for alignment and reconstruction of multiple tilt series in electron tomography. Adv. Struct. Chem. Imaging 2, 8.

Phan, S., Lawrence, A., Molina, T., Lanman, J., Berlanga, M., Terada, M., Kulungowski, A., Obayashi, J., Ellisman, M., 2012. TxBR montage reconstruction for large field electron tomography. J. Struct. Biol. 180, 154164.

Reimer, L., Kohl, H., 2008. Transmission Electron Microscopy Physics of Image Formation, Springer series in optical sciences. Springer Series in Optical Sciences.

Schellenberger, P., Kaufmann, R., Siebert, C.A., Hagen, C., Wodrich, H., Grünewald, 
K., 2014. High-precision correlative fluorescence and electron cryo microscopy using two independent alignment markers. Ultramicroscopy 143, 41-51.

Scott, M.C., Chen, C.-C., Mecklenburg, M., Zhu, C., Xu, R., Ercius, P., Dahmen, U., Regan, B.C., Miao, J., 2012. Electron tomography at 2.4-ångström resolution. Nature 483, 444-447.

Shaikh, T.R., Gao, H., Baxter, W.T., Asturias, F.J., Leith, A., Frank, J., Lourmel, R. De, 2009. Spider. Nat Protoc 3, 1941-1974.

Shu, X., Lev-Ram, V., Deerinck, T.J., Qi, Y., Ramko, E.B., Davidson, M.W., Jin, Y., Ellisman, M.H., Tsien, R.Y., 2011. A genetically encoded tag for correlated light and electron microscopy of intact cells, tissues, and organisms. PLoS Biol. 9.

Suzuki, H., 2002. Sample Distortion Removing Method in Thin Piece Forming. US6417512 B1.

Tang, G., Peng, L., Baldwin, P.R., Mann, D.S., Jiang, W., Rees, I., Ludtke, S.J., 2007. EMAN2: An extensible image processing suite for electron microscopy. J. Struct. Biol. 157, 38-46.

Thomas, J.M., Leary, R., Midgley, P.A., Holland, D.J., 2013. A new approach to the investigation of nanoparticles: Electron tomography with compressed sensing. J. Colloid Interface Sci. 392, 7-14.

van Heel, M., Harauz, G., Orlova, E. V, Schmidt, R., Schatz, M., 1996. A new generation of the IMAGIC image processing system. J. Struct. Biol. 116, 1724.

Ye, K., Lim, L.-H., 2016. Every Matrix is a Product of Toeplitz Matrices. Found. Comput. Math. 16, 577-598. 